Gabriella De Joanna • Filippo M. Santorelli • Carlo Casali Vincenzo Brescia-Morra • Anna Perretti • Lucio Santoro

\title{
Combination of mtDNA mutations in a patient with a mitochondrial multisystem syndrome
}

Received: August 5, 1999 / Accepted: October 5, 1999

\begin{abstract}
We report a patient who manifested a heterogeneous clinical presentation, including hypertrophic cardiomyopathy and hypothyroidism, with initially limited central nervous system involvement, and who harbored the mitochondrial (mt)DNA A3243G mutation. MtDNA analyses also revealed deleted genomes in muscle and blood. This atypical molecular combination may have influenced the clinical phenotype.
\end{abstract}

Key words mtDNA · MELAS genotype-phenotype correlation

\section{Introduction}

Ten years after the first pathogenic mutations in mitochondrial DNA (mtDNA) were discovered, and the concept of "mitochondrial genetics" was introduced into clinical medicine, we count over 50 pathogenic point mutations and rearrangements associated with an array of clinical presentations. However, the issue of genotype-phenotype relationships has become more complex than imagined (De Mauro and Bonilla 1997). We describe a patient with hypertrophic cardiomyopathy and hypothyroidism, but with limited central nervous system involvement, in whom we detected an unexpected combination of mtDNA defects.

F.M. Santorelli $(\square) \cdot$ C. Casali

Istituto di Malattie Nervose e Mentali, La Sapienza University, Viale dell'Università, 30, 00186 Roma, Italy

Tel. +39-06-49914458; Fax +39-06-445-4294

e-mail: fms3@na.flashnet.it.

G. De Joanna $\cdot$ A. Perretti $\cdot$ L. Santoro

Department of Neurophysiopathology, Federico II University,

Napoli, Italy

V. Brecia-Morra

Ospedale Civile, Cava de' Tirreni (SA), Italy

\section{Clinical report and methods}

A 51-year-old woman had suffered from severe hypertrophic cardiomyopathy with arrhythmia and bilateral hearing impairment since her thirties. At age 41 years, hypothyroidism became manifest. Serum free thyroxine (FT) 3 and thyroid stimulating hormone (TSH) levels were reduced. Thyroid autoantibodies were negative. Hyperglycemia was also found, and non-insulin-dependent diabetes mellitus (NIDDM) was confirmed by an oral glucose tolerance test. After informed consent was obtained, muscle biopsy showed decreased cytochrome $c$ oxidase (COX) staining in $10 \%$ of the ragged-red fibers (RRF) found. Biochemically, COX was also reduced (65\% residual activity). Her condition worsened, with generalized muscle weakness and moderate ptosis, but there was no extraocular muscle involvement. Her mental state deteriorated progressively. A brain computed tomography (CT) scan showed bilateral calcifications in the pallidus and thalamus. When she was back at home, the patient suddenly suffered seizures and died of cardiopulmonary arrest.

Her 28-year-old daughter has non-specific ovary and thyroid gland dysfunction, but is otherwise asymptomatic. Neurophysiologic evaluation showed reduced nerve conduction velocities and abnormal visual evoked potentials. The patient's brother is healthy. No other relatives were tested.

\section{Results and discussion}

Clinically, in the patient, a disorder of the type seen in multisystem mitochondrial disorders or in mitochondrial encephalomyopathy, stroke-like episodes, and lactic acidosis (MELAS) was sustained by the morphological and biochemical findings in muscle, and indicated that an mtDNA disorder was likely. Southern blot analysis showed a relatively low proportion (17\%) of the so-called "common" mtDNA deletion in muscle, but not in blood, from the 
A.

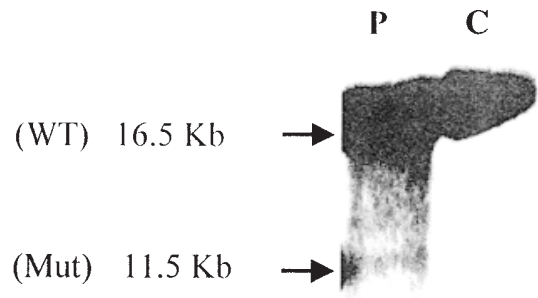

B.

C.
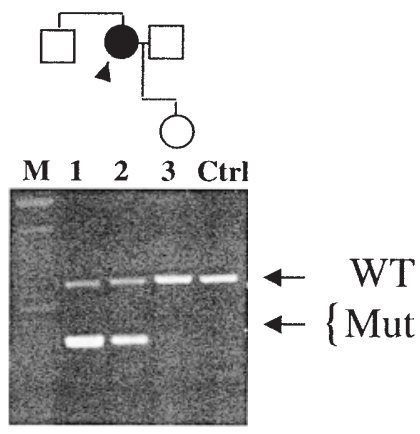

Ctrl

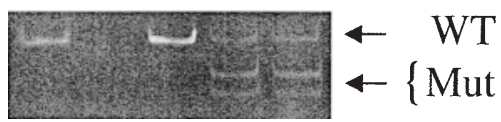

Fig. 1. A Southern blot analysis of muscle from our patient $(P)$ and a control $(C)$ used a standard methodology described in Santorelli et al. (1996). In addition to the wild-type 16.5-kb band, an additional band of approximatively $11.5 \mathrm{~kb}$, corresponding to a deletion of about $5.0 \mathrm{~kb}$, was seen. B The pedigree of this family is illustrated with proposita indicated by filled circle. The strategy to quantitate the "common deletion" of about $5.0 \mathrm{~kb}$, employing oligonucleotide primers $\left(5^{\prime}-3^{\prime}\right) 8200$ $8225 \mathrm{~F}, 9030-9055 \mathrm{~B}$, and $13530-13500 \mathrm{~B}$, is also shown. A wild-type fragment of 850-bp was found in the patient's muscle and blood (lanes 1 and 2), her daughter's lymphocytes (lane 3), and in control (Ctrl) muscle and blood. An additional, mutated fragment was detected only in the patient. C Autoradiogram showing the mutant load of the A $3243 \mathrm{G}$ mutation. The 238-base pair (bp) polymerase chain reaction (PCR) amplified fragment is normally cleaved by the endonuclease HaeIII in three fragments of 169,37 , and $32 \mathrm{bp}$. The A3243G mutation creates an additional restriction site, producing 72-bp and 97-bp fragments. The levels of the A3243G mutation were determined using a reported radioactive methodology (Ciafaloni et al. 1992). WT, Wildtype; Mut, mutant mtDNA; $M$, molecular marker size

proposita (Fig. 1A). The "common" deletion of about $5 \mathrm{~kb}$ accounted for $47 \%$ of total genomes in blood and $67 \%$ of total genomes in muscle when assessed using a sensitive three-primer polymerase chain reaction (PCR) method, as described (Sciacco et al. 1994) (Fig. 1B). We also found the A3243G mutation in blood and muscle mtDNA in our proposita in similar percentages $(55 \%)$, as well as detected this mutation in lymphocytes from her daughter (Fig. 1C), who did not harbor deletions. The unusual clinical and molecular combination suggested that additional factors may have determined the phenotype. We hypothesized that other alterations affecting genes important for protein translation or impairing energy production could account for the more severe clinical phenotype in the mother, and we sequenced $80 \%$ of mtDNA in the proposita and in two maternal relatives. We found no variations of pathogenic significance in the tRNAs and rRNA-coding sequences, whereas we identified several changes in the two adenosine triphosphatase (ATPase) subunits, but most had already been reported in the normal population (Mitomap 1999). We also found a new, homoplasmic T9137C mutation in the patient, but not in 50 control subjects: the mutation converts a moderately conserved isoleucine by tryptophan but its pathogenic role is still unclear.

The typical manifestations of the A3243G mutation include extraocular and skeletal myopathy, with RRF, lactic acidosis, encephalopathy with stroke-like episodes, NIDDM and deafness, and cardiomyopathy. However, in affected families, most carriers are either asymptomatic or present with different combinations of symptoms, such as hearing loss, cardiomyopathy, or NIDDM. An endocrine disorder such as hypothalamic growth hormone deficiency or delayed puberty may also occur (Manouvrier et al. 1995; Yang et al. 1995). Hypothyroidism, which was observed in our patient, could be an underestimated manifestation (Manouvrier et al. 1995). Interestingly, a Japanese patient with hypothyroidism and features of the Kearns-Sayre syndrome has been reported to harbor a similar "double" mtDNA mutation (Ohno et al. 1996).

Differences between levels of mutant mtDNA in organs and tissues within the same individual are often considered to be the principal factor responsible for the varied clinical expression of the mtDNA defect. It has been shown that most, but not all, A3243G individuals harbor more abundant mutant genomes in muscle than in blood, and that clinical involvement correlates well with mutant load in muscle but not in blood, with higher percentages of the mutated mtDNA resulting in severe involvement of the central nervous system (Chinery et al. 1997). In our patient, intermediate levels of mutant A3243G were detected, and this probably determined the relatively milder phenotype. We cannot exclude the possibility that different tissues, other than blood and muscle, harbored higher percentages of mutated mtDNAs. Also, we assessed levels of the mutations at a particular time point and it is uncertain whether the aging process influences the severity of presentation and the mutant load. For instance, this influence has been noted in some mtDNA tRNA mutations (Fu et al. 1996; Weber et al. 1997).

In conclusion, we suggest that, in patients with mitochondrial DNA defects, signs and symptoms in the patient be considered by the appropriate internist, as these manifestations may precede the development of a central nervous system disorder.

Acknowledgments We are indebted to Mrs. Tracey L. PeriouReinberg for revising the manuscript. Telethon-Italia Grant n. 844 is gratefully acknowledged.

\section{References}

Chinnery PF, Howell N, Lightowlers RN, Turnbull DM (1997) Molecular pathology of MELAS and MERRF. The relationship be- 
tween mutation load and clinical phenotypes. Brain 120:1713-1721

Ciafaloni E, Ricci E, Shanske S, Moraes CT, Silvestri G, Hirano M, Shimonetti S, Angelini C, Donati MA, Garcia C, DiMauro S (1992) MELAS: clinical features, biochemistry, and molecular genetics. Ann Neurol 31:391-398

DiMauro S, Bonilla E (1997) Mitochondrial encephalomyopathies. In: Rosenberg R, Prusiner S, DiMauro S, Barchi R (eds) The molecular and genetic basis of neurological disease. Butterworth-Heinemann, Boston, pp 201-235

Fu K, Hartlen R, Johns T, Genge A, Karpati G, Shoubridge EA (1996) A novel heteroplasmic tRNA Leu(CUN) mtDNApoint mutation in a sporadic patient with mitochondrial encephalomyopathy segregates rapidly in skeletal muscle and suggests an approach to therapy. Hum Mol Genet 5:1835-1840

Manouvrier S, Rotig A, Hannebique G, Gheerbrandt JD, RoyerLegrain G, Munnich A, Parent M, Grunfeld JP, Largilliere C, Lombes A (1995) Point mutation of the mitochondrial $t R N A$ (Leu) gene $(\mathrm{A} 3243 \mathrm{G})$ in maternally inherited hypertrophic cardiomyopathy, diabetes mellitus, renal failure, and sensorineural deafness. J Med Genet 1995; 32:654-656

MITOMAP: a human mitochondrial genome database. Center for Molecular Medicine, Emory University, Atlanta, GA, USA, 1999;
(URL:http://www.gen.emory.edu/mitomap.html)

Ohno K, Yamamoto M, Engel AG, Harper CM, Roberts LR, Tan GH, Fatourechi V (1996) MELAS- and Kearns-Sayre-type co-mutation with myopathy and autoimmune polyendocrinopathy. Ann Neurol 39:761-766

Santorelli FM, Sciacco M, Tanji K, Shanske S, Vu TH, Golzi V, Griggs RC, Mendell JR, Hays AP, Bertorini TE, Pestronk A, Bonilla E, DiMauro S (1996) Multiple mitochondrial DNA deletions in sporadic inclusion body myositis: a study of 56 patients. Ann Neurol 39:789-795

Sciacco M, Bonilla E, Schon EA, DiMauro S, Moraes CT (1994) Distribution of wild-type and common deletion forms of mtDNA in normal and respiration-deficient muscle fibers from patients with mitochondrial myopathy. Hum Mol Genet; 3:13-19

Weber K, Wilson JN, Taylor L, Brierley E, Johnson MA, Turnbull DM, Bindoff LA (1997) A new mtDNA mutation showing accumulation with time and restriction to skeletal muscle. Am J Hum Genet 60:373-380

Yang CY, Lamm HC, Lee HC, Wei YH, Lu CC, Han TM, Tsai JL, Chuang YH, Lee JK (1995) MELAS syndrome associated with diabetes mellitus and hyperthyroidism: a case report from Taiwan. Clin Endocrinol 43:235-239 